\title{
The Patched 1 Tumor-Suppressor Gene Protects the Mouse Lens from Spontaneous and Radiation-Induced Cataract
}

\author{
Ilaria De Stefano, ${ }^{*}$ Barbara Tanno, ${ }^{\dagger}$ Paola Giardullo, ${ }^{*}$ Simona Leonardi, ${ }^{\dagger}$ Emanuela Pasquali, ${ }^{\dagger}$ Francesca Antonelli, \\ Mirella Tanori, ${ }^{\dagger}$ Arianna Casciati, ${ }^{\dagger}$ Simonetta Pazzaglia, ${ }^{\dagger}$ Anna Saran, ${ }^{\dagger}$ and Mariateresa Mancuso ${ }^{\dagger}$
}

From the Department of Radiation Physics, * Guglielmo Marconi University, Rome; and the Laboratory of Radiation Biology and Biomedicine, ${ }^{\dagger}$ Agenzia Nazionale per le Nuove Tecnologie, l'Energia e lo Sviluppo Economico Sostenibile (ENEA), Rome, Italy

\author{
Accepted for publication \\ September 4, 2014. \\ Address correspondence to \\ Anna Saran, Ph.D., or \\ Mariateresa Mancuso, Ph.D., \\ Laboratory of Radiation \\ Biology and Biomedicine, \\ ENEA, 00123 Rome, Italy. \\ E-mail: anna.saran@enea.it or \\ mariateresa.mancuso@enea.it.
}

\begin{abstract}
Age-related cataract is the most common cause of visual impairment. Moreover, traumatic cataracts form after injury to the eye, including radiation damage. We report herein that sonic hedgehog (Shh) signaling plays a key role in cataract development and in normal lens response to radiation injury. Mice heterozygous for Patched 1 (Ptch1), the Shh receptor and negative regulator of the pathway, develop spontaneous cataract and are highly susceptible to cataract induction by exposure to ionizing radiation in early postnatal age, when lens epithelial cells undergo rapid expansion in the lens epithelium. Neonatally irradiated and control Ptch $1^{+/-}$mice were compared for markers of progenitors, Shh pathway activation, and epithelialto-mesenchymal transition (EMT). Molecular analyses showed increased expression of the EMT-related transforming growth factor $\beta /$ Smad signaling pathway in the neonatally irradiated lens, and upregulation of mesenchymal markers Zeb1 and Vim. We further show a link between proliferation and the stemness property of lens epithelial cells, controlled by Shh. Our results suggest that Shh and transforming growth factor $\beta$ signaling cooperate to promote Ptch1-associated cataract development by activating EMT, and that the Nanog marker of pluripotent cells may act as the primary transcription factor on which both signaling pathways converge after damage. These findings highlight a novel function of Shh signaling unrelated to cancer and provide a new animal model to investigate the molecular pathogenesis of cataract formation. (Am J Pathol 2015, 185: 85-95; http://dx.doi.org/10.1016/j.ajpath.2014.09.019)
\end{abstract}

Cataract is the most frequent cause of blindness worldwide. Development of cataract in adults is related to normal aging, but other factors are also involved, such as diabetes, smoking, and alcohol use, as well as exposure to UVB and ionizing radiations. ${ }^{2}$ Cataract is also one of the most important causes of avoidable (preventable and treatable) childhood blindness, ${ }^{3}$ and often develops as a complication of radiotherapy. ${ }^{4}$ However, the exact mechanisms of radiogenic cataract are not entirely understood.

Inherited mutations of the Patched 1 (Ptchl) gene have been identified as responsible for Gorlin syndrome. ${ }^{5}$ Patients with this hereditary condition are hypersensitive to radiation and prone to develop multiple skin cancers, specifically basal cell carcinomas, ${ }^{6}$ occurring with greater incidence in portals of radiotherapy. ${ }^{7}$ They are also at risk for developing medulloblastoma, a specific type of brain tumor. Among many minor criteria for diagnosis, ophthalmic abnormalities, including cataract, occur in $26 \%$ of patients. ${ }^{8,9}$

Mice in which one copy of Ptchl is inactivated are characterized by activation of the sonic hedgehog (Shh) pathway and show increased susceptibility to spontaneous and radiationinduced tumors, providing an ideal in vivo model for studying the typical pathological conditions associated with Gorlin syndrome.$^{10-12}$ In addition to the well-known role of hedgehog signaling in cancer, Shh signals direct cell proliferation, cell fate determination, epithelial-to-mesenchymal transition (EMT), and the rearrangement of cells by motility and adhesion changes

Supported in part by the Unit of Radiation Biology and Human Health, l'Energia e lo Sviluppo Economico Sostenibile, Intramural Program (M.M and A.S.).

I.D.S., B.T., and P.G. contributed equally to this work.

Disclosures: None declared. 
during embryogenesis, remaining largely expressed in the stem cell compartment of adult tissues. ${ }^{13}$ The vertebrate ocular lens, composed of epithelial and fiber cells, grows throughout life, implying the existence of a lens stem cell compartment. DNA labeling studies showed that most new lens cells arise in the germinative zone, a narrow cellular region that rings the lens epithelium toward the periphery of the anterior lens surface. ${ }^{14,15}$ After division, these cells withdraw from the cell cycle and terminally differentiate into fiber cells. Despite findings showing a role of Shh in mouse lens development ${ }^{16}$ and in lens regeneration of the adult newt, ${ }^{17}$ much remains to be understood about the role of this pathway in postnatal lens growth and its possible correlation with cataract development.

Herein, we show a specific role for the Ptchl gene in the maintenance of lens integrity with or without exogenous damage, and validate the $P t c h 1^{+/-}$model as a tool to understand the molecular mechanism of cataract development. Altogether, our results support the hypothesis that Shh and transforming growth factor $\beta$ (TGF- $\beta$ ) signaling pathways synergize with radiation damage to induce EMT, culminating in anterior subcapsular cataract (ASC) development. We also outline a role of the Nanog stemness gene in Ptchl-associated radiogenic cataract.

\section{Materials and Methods}

Mice

Mice lacking one Ptchl allele (Ptch1 $1^{+ \text {neo6/7 }}$; named Ptchl $^{+/-}$throughout the text) on CD1 background were bred and genotyped as described. ${ }^{10}$ Care of experimental animals was in accordance with the Italian legislation on animal experimentation. Experimental protocols were reviewed by the Institutional Animal Care and Use Committee.

\section{Mice Irradiation and Monitoring}

Ptch $^{+/-}$and wild-type (WT) littermates of both sexes were irradiated using a Gilardoni CHF 320 G X-ray generator
(Gilardoni, Mandello del Lario, Italy), as described. ${ }^{12}$ Animals were exposed to a single dose of $3 \mathrm{~Gy}$ at different postnatal $(\mathrm{P})$ days (P2, P10, P56). Additional groups were left untreated as controls. Experimental groups are summarized in Table 1.

Mice were observed daily throughout their life span. The time of cataract onset (unilateral and bilateral) (Table 1) was recorded. On decline of health, mice were sacrificed and necropsied.

\section{Histological, IHC, and Morphometric Analyses}

Normal and cataract-bearing eyes were processed for histological analysis by standard techniques at the end of their lifetime or when bilateral cataract became evident; eyes were also collected from mice sacrificed before termination of the experiment. Eye sections ( $2 \mu \mathrm{m}$ thick) were cut in a plane perpendicular to the anteroposterior eye axis and stained with hematoxylin and eosin. Immunohistochemistry (IHC) was performed on the following groups: i) eyes from satellite groups ( $n=6$ per genotype) irradiated at $\mathrm{P} 2$ and sacrificed at short-term after irradiation (ie, 0.5 hours for analysis of $\gamma-\mathrm{H} 2 \mathrm{AX}$ foci (a biomarker of DNA double-strand breaks), 4.5 hours for detection of p53 and p21 immunoreactivity, and 6 hours for apoptotic analysis by activated caspase-3 antibody); ii) eyes from unirradiated mice at $\mathrm{P} 2, \mathrm{P} 4, \mathrm{P} 6$, and $\mathrm{P} 10$ or $\mathrm{P} 2$-irradiated mice sacrificed at different times after irradiation ( $\mathrm{P} 4, \mathrm{P} 6$, and P10) for Ki-67 proliferation analysis and nestin immunoreactivity; and iii) fully developed cataracts. The following antibodies were used: Ki-67 (NCL-Ki67p; polyclonal; dilution 1:800; Novocastra Laboratories, Newcastle, UK); $\gamma$-H2AX (05-636; monoclonal; dilution 1:200; Upstate Biotechnology Inc., Lake Placid, NY); cleaved caspase-3 (9661; polyclonal; dilution 1:100; Cell Signaling Technology, Inc., Danvers, MA); E-cadherin (3195; polyclonal; dilution 1:200; Cell Signaling Technology, Inc.); N-cadherin (13116; polyclonal; dilution 1:200; Cell Signaling Technology, Inc.); anti-nestin (ab81755; polyclonal; dilution 1:300; Abcam, Cambridge, UK); $\alpha$-smooth muscle actin (A2547;

Table 1 Incidence of Cataract in $P t c h 1^{+/-}$and Ptch1 ${ }^{+/+}$Mice Irradiated at 2, 10, and 56 Days of Age with 3 Gy of X-Rays or Left Unirradiated

\begin{tabular}{|c|c|c|c|c|c|c|}
\hline Treatment & Age, days & Mouse line & No. of mice & $\begin{array}{l}\text { Unilateral cataract, } \\
\% \text { (no./total) }\end{array}$ & $\begin{array}{l}\text { Bilateral cataract, } \\
\% \text { (no./total) }\end{array}$ & $\begin{array}{l}\text { Median latency, } \\
\text { weeks }\end{array}$ \\
\hline 3 Gy & 2 & Ptch1 $1^{+/-}$ & 42 & $45.2(19 / 42)^{* \dagger}$ & $14.3(6 / 42)^{\ddagger}$ & 6.9 \\
\hline \multirow[t]{2}{*}{3 Gy } & 10 & Ptch $1^{+/-}$ & 33 & 0 & 0 & - \\
\hline & & Ptch $1^{+/+}$ & 47 & 0 & 0 & - \\
\hline 3 Gy & 56 & $\operatorname{Ptch}^{+/-}$ & 44 & $2.27(1 / 44)$ & 0 & 7 \\
\hline & & Ptch1 $^{+/+}$ & 53 & 0 & 0 & - \\
\hline
\end{tabular}

All $P$ values were calculated using Fisher's exact test.

${ }^{*} P<0.001$ between unilateral cataract P2 irradiated $P$ tch $1^{+/-}$mice and unirradiated $P$ tch ${ }^{+/-}$mice.

${ }^{\dagger} P<0.0001$ between unilateral cataract $\mathrm{P} 2$ irradiated $P$ tch $1^{+/-}$and $P \operatorname{tch} 1^{+/+}$mice.

${ }^{\ddagger} P<0.05$ between bilateral cataract $\mathrm{P} 2$ irradiated $P$ tch $1^{+/-}$and $P$ tch $1^{+/+}$mice.

${ }^{\S} P<0.05$ between unilateral cataract $P 2$ irradiated $P$ tch $1^{+/+}$mice and unirradiated $P$ tch $1^{+/+}$mice.

Ptch1, Patched 1. 
dilution 1:400; Sigma-Aldrich, St. Louis, MO); p21 (ab2961; polyclonal; dilution 1:100; Abcam); and p53 (NCL-p53CM5p; polyclonal; dilution 1:500; Novocastra Laboratories).

The IHC scoring (proliferation and apoptotic indexes) was performed by investigators blinded to treatment groups (I.D.S. and P.G.). The number of cells positive for Ki-67 or cleaved caspase-3 antibody was calculated as percentage of total lens epithelial cells (LECs). Nestin IHC expression intensity was assessed by HistoQuest automated IHC analysis system (TissueGnostics, Vienna, Austria).

Morphometric analysis of lens capsule in mild and severe cataract was performed on high-magnification digital images $(n=6)$. Capsule thickness was recorded using imaging software NIS-Elements BR version 4.00 .05 (Nikon Instruments Europe B.V., Amsterdam, the Netherlands), and averages were calculated from approximately 400 measurements.

Lens area was determined from $P t c h 1^{+/-}$and WT mice ( $n=6$ per genotype) at 21 days of age using imaging software NIS-Elements BR 4.00 .05 and reported as means $\pm \mathrm{SD}$.

\section{Real-Time Quantitative PCR}

Mice of each genotype $(n=6)$ were left unirradiated and sacrificed at $\mathrm{P} 2$ or P10, or they were irradiated at P2 and sacrificed after 8 days. Eyes were enucleated, and lenses were removed and snap frozen in liquid nitrogen before storing at $-80^{\circ} \mathrm{C}$.

Total RNA was isolated using RNeasy Mini Kit (Qiagen, Milan, Italy). Purified RNA was quantified using a NanoDrop spectrophotometer (Thermo Fisher Scientific Inc., Milan, Italy), and $1 \mu \mathrm{g}$ of total RNA was reverse transcribed with High-Capacity cDNA Reverse Transcription Kit (Applied Biosystems, Foster City, CA), then stored at $-20^{\circ} \mathrm{C}$ until use. Primers were designed for nine target genes working under the same cycling conditions (Table 2). Real-time quantitative PCR (qPCR) was performed by StepOnePlus Real-Time PCR System (Applied Biosystems) using Power SYBR Green PCR Master Mix (Applied Biosystems). Reactions were performed in triplicate. Relative gene expression was quantified using glyceraldehyde-3-phosphate dehydrogenase as housekeeping gene. The standard curve method was used to normalize expression of the reference gene and to calculate the relative expression levels of target genes.

\section{Western Blot Analysis}

Proteins were extracted from lens pools $(n=12)$ of unirradiated Ptchl ${ }^{+/-}$and WT mice at P2 and P10, and of mice irradiated at P2 and sacrificed after 8 days, then analyzed as described. ${ }^{18}$ After incubation with anti-Smad3 phospho-S423/S425 (ab51451; polyclonal; dilution 1:1000; Abcam) and $\beta$-actin (A5441; monoclonal; SigmaAldrich), specific proteins were visualized with ImageQuant LAS 500 system (GE Healthcare Europe GmbH, Milan, Italy).

\section{Statistical Analysis}

Cataract incidence, expressed as the percentage of mice with macroscopic unilateral cataract, was analyzed using a Fisher's exact test. Kaplan-Meier survival curves were compared, and log-rank test $P$ values were calculated. IHC scoring, intensity, and morphometric and qPCR analyses are reported as means $\pm \mathrm{SD}$, and a $t$-test was used for determination of statistical difference between groups. $P \leq 0.05$ was considered statistically significant. Analyses were performed using GraphPad Prism version 5.0 for Windows (GraphPad Software, San Diego, CA).

\section{Results}

\section{Ptch1 Deficiency Causes Cataract}

To test the role of Shh signaling pathway deregulation in cataract development, Ptch1 ${ }^{+/-}(n=34)$ and WT $(n=$ 53) mice were observed throughout their life span. Ptch1 ${ }^{+/-}$mice developed grossly visible cataract, recognizable as a white pinpoint focus in unpigmented eyes, with an incidence of $8.8 \%(3 / 34)$ and median latency of 34 weeks (Figure 1, A and B). The event was bilateral in twothirds of positive mice. No cataract development was observed in WT mice $(0 / 53 ; P=0.05)$ (Figure $1 \mathrm{C}$ and Table 1), showing a specific role for Ptchl in the preservation of lens integrity.

Table 2 Primer Sequences Used for Real-Time Quantitative PCR

\begin{tabular}{|c|c|c|}
\hline Gene & Forward primer & Reverse primer \\
\hline Gli1 & $5^{\prime}$-GAGGACCTGGAGAGAGAGGAGAA-3' & $5^{\prime}$-CCAGCGGCAGTCTGTCTCA-3' \\
\hline Gli2 & $5^{\prime}-$ CGCAAGCGGGCTCTGT-3' & 5'-TCCGAATCATGCGTTGTAGGT-3' \\
\hline Vim & $5^{\prime}-$ GAGAGAGGAAGCCGAAAGCA-3' & $5^{\prime}$-GCCAGAGAAGCATTGTCAACATC-3' \\
\hline Cdh1 (E-cadherin) & $5^{\prime}-\mathrm{AGAGCCGGACACGTTCATG}-3^{\prime}$ & $5^{\prime}-$ TTGGCAGTGTCCCTCCAAA-3' \\
\hline Pax6 & $5^{\prime}$-CCATGTTGGGCCGAACA-3' & 5'-TGGGTGGCAAAGCACTGTAC-3' \\
\hline Gapdh & 5'-CATGGCCTTCCGTGTTCCTA-3' & $5^{\prime}-$ GCGGCACGTCAGATCCA-3' \\
\hline
\end{tabular}



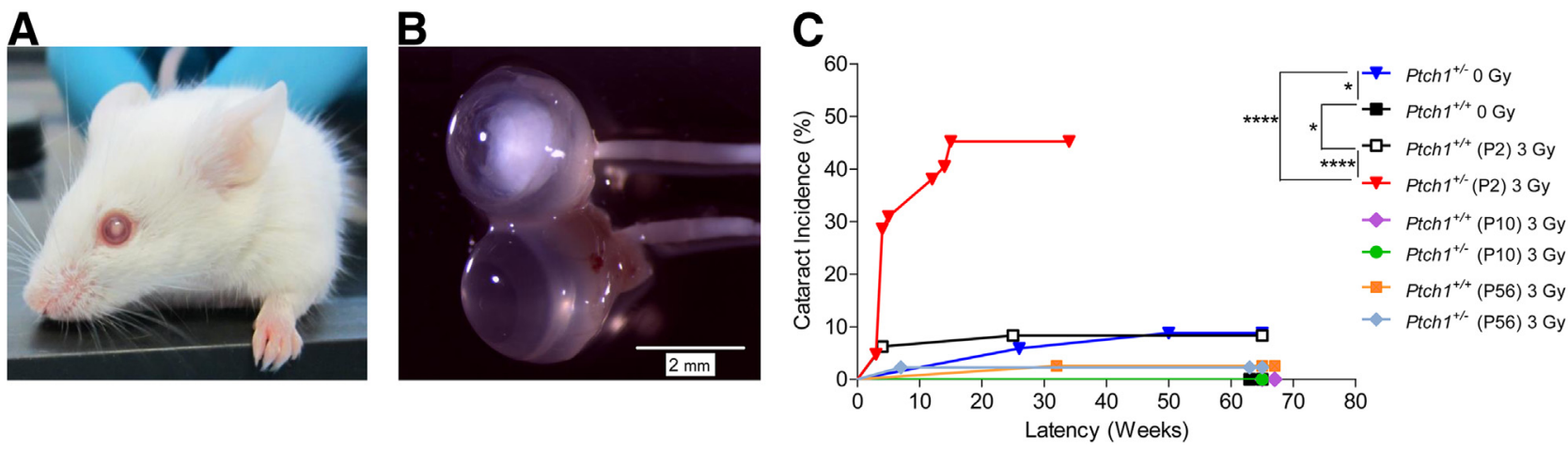

Figure 1 Cataract development in Patched $1\left(P t c h 1^{+/-}\right)$and wild-type (WT) mice after irradiation at P2. A: Macroscopically visible cataract is recognizable as a white pinpoint focus in the pink eyes. B: Stereomicroscopic image of eyes showing the typical lens opacity associated with cataract compared to normal lens appearance. C: Incidence of cataract in irradiated and unirradiated $P \operatorname{tch} 1^{+/-}$and WT mice. ${ }^{*} P<0.05,{ }^{* * *} P<0.0001$.

\section{Ptch1 Deficiency Confers Increased Susceptibility to Radiogenic Cataract Mainly in the Early Stages of Postnatal Lens Morphogenesis}

$P t c h 1^{+/-}$mice display many characteristics associated with Gorlin syndrome, including hypersensitivity to ionizing radiation. To test whether ionizing radiation can be an inducer of cataract in this model, groups of $\mathrm{Ptchl}^{+/-}$and WT mice were whole-body irradiated with 3 Gy of X-rays at different postnatal ages (ie, at P2, an early stage of postnatal lens development; at P10, when postnatal development is near to completion; and at P56, in young adults). Results show low or no induction of cataract when radiation was delivered at P10 or P56, irrespective of Ptchl status (Table 1). In contrast, Ptchl ${ }^{+-}$mice irradiated at P2 showed a high cataract incidence compared to unirradiated Ptch $1^{+/-}$mice $(P<0.0001)$. Of 42 mice, $19(45.2 \%)$ developed cataract, and the event was bilateral in $6(14.3 \%)$ of 19 positive mice. Notably, in 12 of 19 positive mice, cataract development was an extremely early event, detected at 3 weeks of age, although the total median latency was 6.9 weeks (Figure 1C and Table 1). At a highly radiosensitive P2 age, $8.3 \%$ of WT mice also developed cataract with median latency of 9.5 weeks. These data demonstrate that germline inactivation of one copy of Ptchl confers increased predisposition to radiation-induced cataract, with high susceptibility during the early stages of postnatal lens morphogenesis.

\section{Radiation and Shh Deregulation Synergize to Enhance EMT}

Histopathological and IHC analyses showed no differences between normally appearing lenses of adult Ptchl $^{+/-}$and WT mice (Figure 2, A-D). A single layer of cuboidal cells covering the anterior lens surface, the LECs (Figure 2A), showed high immunoreactivity for the adhesion molecule E-cadherin (Figure 2B). LECs showed lack of N-cadherin signal (Figure 2C), consistent with the specificity of this marker for fiber cells. ${ }^{19}$
All cataracts (spontaneous or induced by radiation in WT and in $\mathrm{Ptchl}^{+/-}$mice) had similar morphological characteristics at histological examination (ie, they had histological features of ASC with different degrees of damage severity). In milder cases, LECs were characterized by loss of epithelial cell polarity (Figure 2, E and F), prominent cell vacuolization (Figure 2E), and coexistence of E-cadherin (Figure 2F) and $\mathrm{N}$-cadherin (Figure 2G) expression, indicative of EMT activation in lens epithelium (Figure 2, $\mathrm{C}$ and $\mathrm{G}$ ). In addition, disorganization of fiber cell structure was already evident (Figure 2, E and H). In more severe disease, LECs formed a plaque consisting of multilayered cells with spindle-shaped features, reminiscent of fibroblastic morphological features (Figure 2I). IHC analyses showed limited reactivity for E-cadherin and $\mathrm{N}$-cadherin (Figure 2, J and K). Aberrant deposition of collagen was evident (Figure 2L), as was expression of the EMT marker, $\alpha$-smooth muscle actin (Figure 2M). At this stage, lens fiber cells were dramatically compromised (Figure 2M). Capsule thickness correlated with disease severity (Figure 3, A-D). In milder cases, there was a twofold increase compared to normal lens $(5.4 \mu \mathrm{m}$ versus $2.6 \mu \mathrm{m} ; P<0.0001)$ (Figure 3 , $\mathrm{A}$ and $\mathrm{B})$. This trend was confirmed in severe cataracts, in which average capsule thickness was sixfold compared to normal lens $(14 \mu \mathrm{m}$ versus $2.6 \mu \mathrm{m} ; P<0.0001)$ (Figure 3, $\mathrm{A}$ and $\mathrm{C})$. Altogether, these data allow classification of cataracts observed as ASCs, developing through a mechanism guided by EMT.

\section{Shh Deregulation Drives LECs toward a More Proliferative and Undifferentiated Condition}

During lens embryonal development, all LECs have been shown to actively proliferate. ${ }^{20}$ However, during the first postnatal week, the lens is still in rapid growth, the number of mitotically active LECs changes profoundly, with significantly increased dividing cells between P1 and P3, and a decline is observed at P5. ${ }^{21}$

We evaluated the proliferative capacity of the LECs in Ptch $^{+/-}$and WT mice at P2, the age of peak susceptibility 

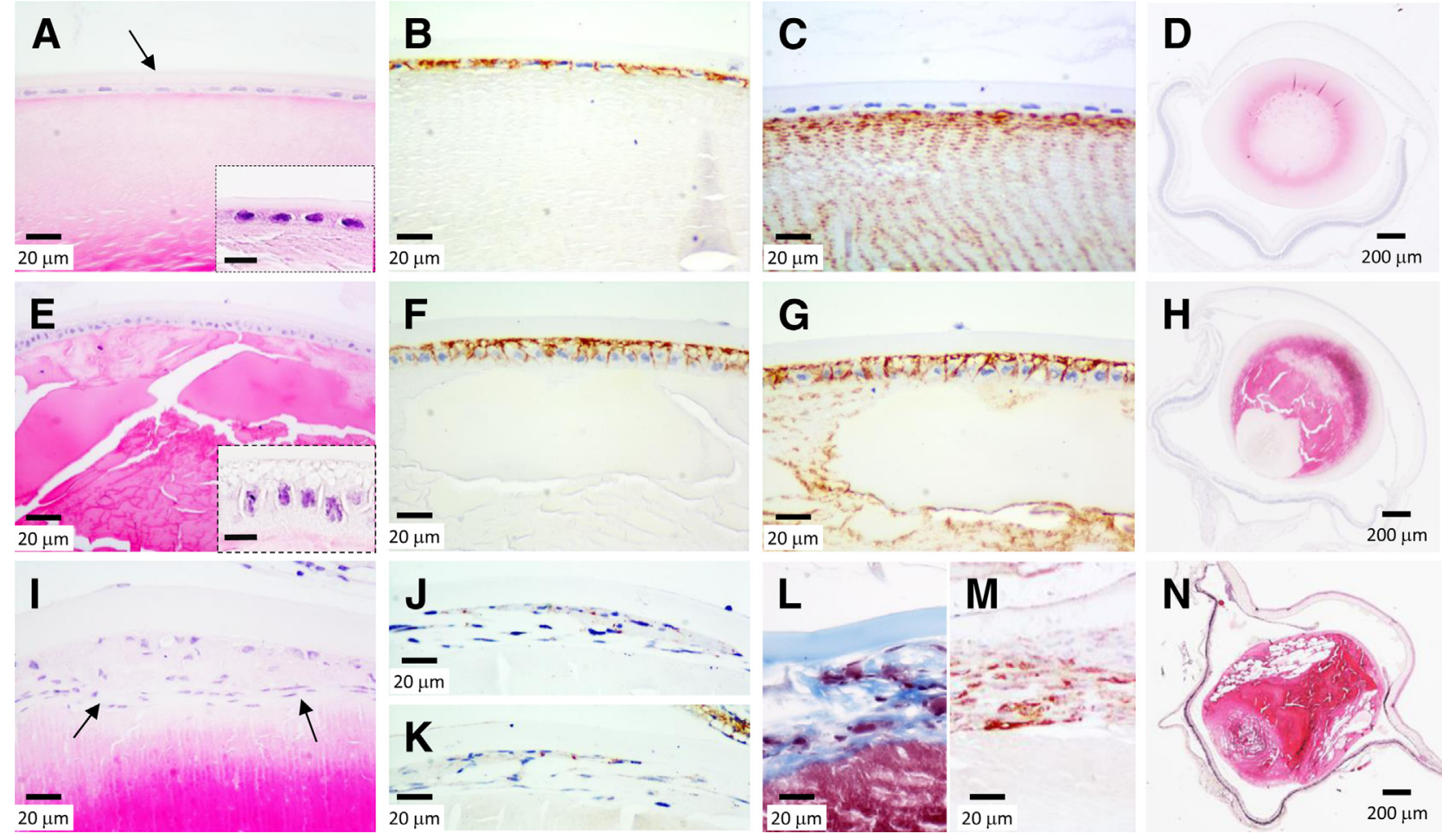

Figure 2 Patched $1\left(P t c h 1^{+/-}\right)$and wild-type (WT) mice develop anterior subcapsular cataract (ASC). A-D: Representative images of lens epithelial cells (LECS) of a normal unirradiated mouse lens. A: Histological image of the typical simple cuboidal epithelium, showing strong E-cadherin immunoreactivity (B), and lack of $\mathrm{N}$-cadherin expression (C). The arrow in $\mathbf{A}$ indicates the capsule. D: Representative image of a normal unirradiated mouse lens. E: Early-stage cataracts show vacuolated and elongated LECs and concomitance of E-cadherin (brown stain; F) and N-cadherin (brown stain; G) immunoreactivity. H: The typical disorganization of fiber cell structure is already evident. I: Severe ASC, characterized by a typical multilayered plaque (arrows) consisting of spindleshaped cells, lack of E-cadherin ( $\mathbf{J}$ ) and $\mathrm{N}$-cadherin (K) expression, deposition of collagen fibers (blue stain), as demonstrated by Masson's trichrome staining (L), and typical $\alpha$-smooth muscle actin expression (red stain; M). N: Extensive disorganization of fiber cells. Scale bar $=10 \mu \mathrm{m}(\mathbf{A}$ and $\mathbf{E}$, insets).

to radiation injury, and at $\mathrm{P} 10$, a nonresponsive age. By using a Ki-67 proliferation marker, we show that at P2, many LECs are mitotically active (Figure 4, A and B). In contrast, at P10, only a few centrally located LECs are Ki-67 positive (Figure 4, C and D), confirming an age-related decline of proliferation in both WT and Ptch1 ${ }^{+/-}$LECs. To explore potential differences due to Ptchl allelic status, Ki-67positive cells were quantified at $\mathrm{P} 2, \mathrm{P} 4, \mathrm{P} 6$, and P10 in WT and Ptch $^{+/-}$mice. Figure 4E shows that the proliferation index of the LECs in Ptchl $^{+/-}$mice was significantly higher compared to WT mice at $\mathrm{P} 2, \mathrm{P} 4$, and $\mathrm{P} 6$. There were more Ki67-positive cells in the Ptch1 ${ }^{+/-}$lens at P10, although this difference was not statistically significant. This evidence suggests that the Shh pathway may mediate cell proliferation during lens development by acting as a mitogen, similar to its role in other developing tissues/organs (eg, the cerebellum). ${ }^{22}$

Because $P t c h 1^{+/-}$mice have increased LECs proliferation, we investigated whether their lenses are larger compared to WT mice. We performed morphometric analyses to measure lens area on sections from unirradiated $\mathrm{Ptchl}^{+/-}$and WT
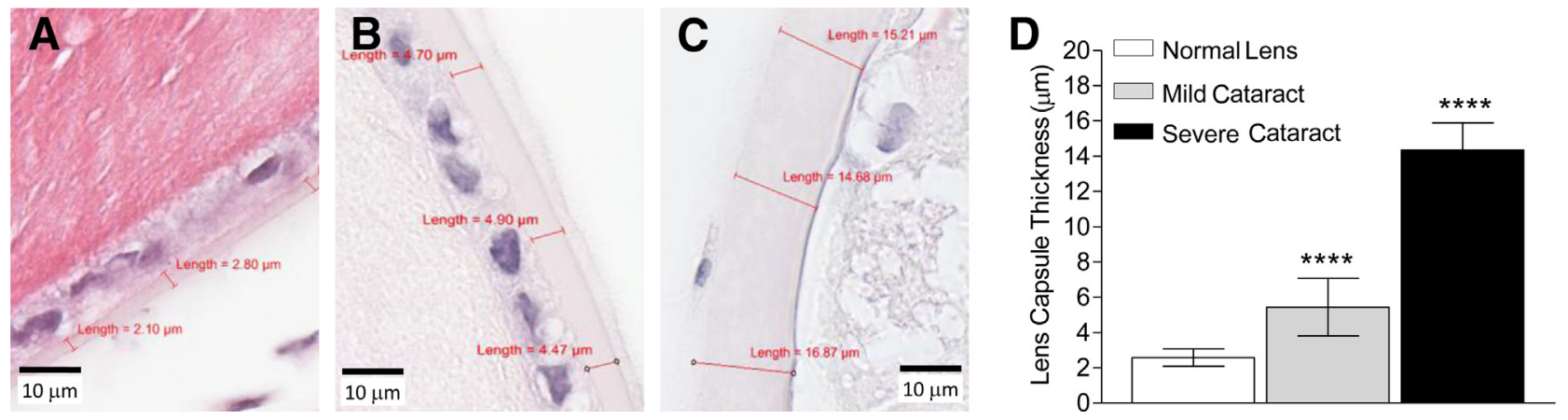

Figure 3 Representative images of the capsule thickness in normal lens (A), mild anterior subcapsular cataract (ASC; B), and severe ASC (C), with quantification (D). ${ }^{* \star * *} P<0.0001$. 

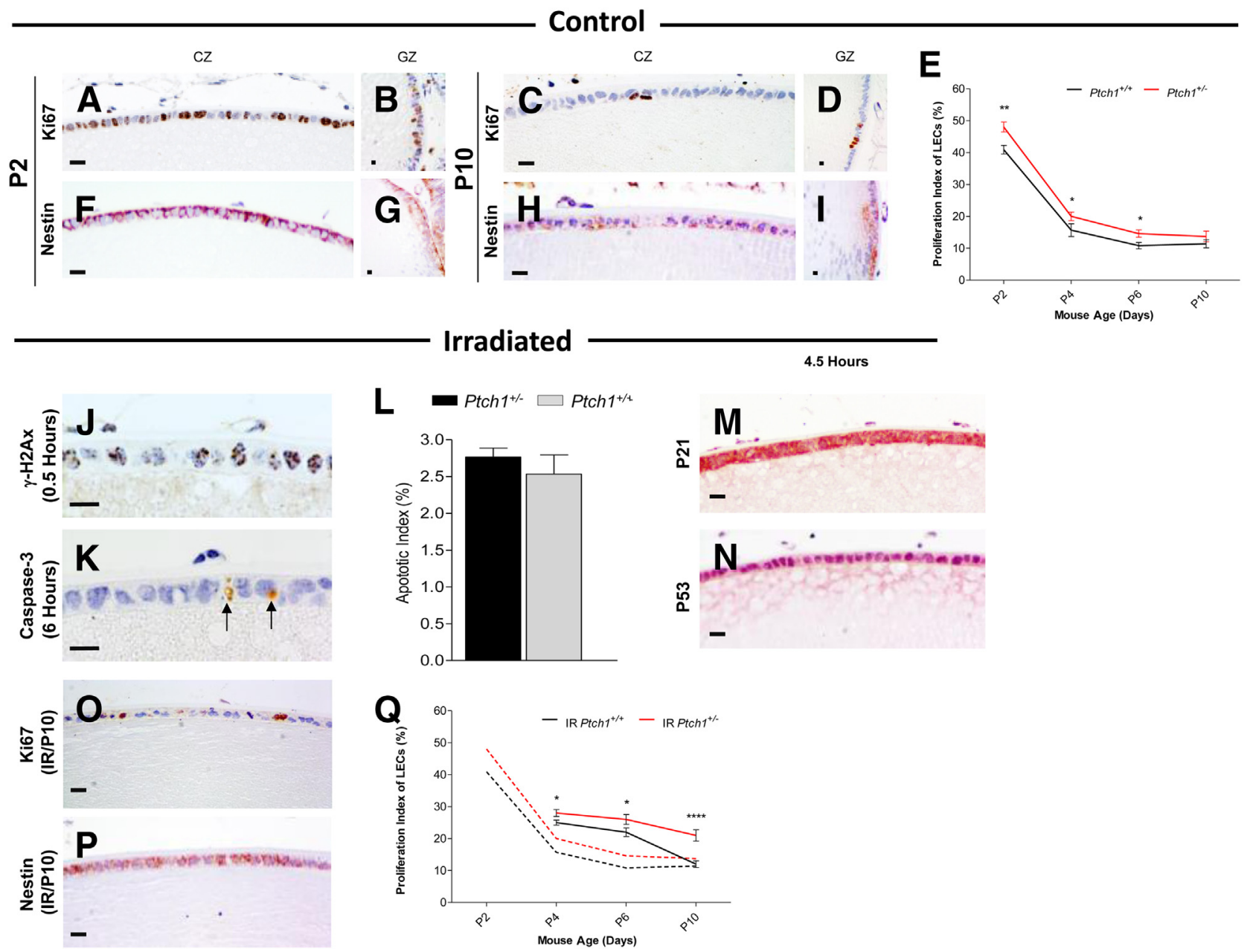

Figure 4 Sonic hedgehog drives lens epithelial cells (LECS) toward a more proliferative and undifferentiated condition. Representative images of unexposed Patched $1\left(\right.$ Ptch $\left.^{+/-}\right)$LECs after immunostaining with an antibody direct against Ki-67 at P2 (A and $\left.\mathbf{B}\right)$ and P10 (C and D). E: Kinetics of proliferation of unirradiated Ptch $1^{+/-}$and wild-type (WT) mouse LECs. Representative images of Ptch $1^{+/-}$LECs immunostained at P2 (F and G) and P10 (H and I) with antinestin antibody. Evaluation of DNA damage response using an antibody directed against $\gamma$-H2AX (J) or activated caspase-3 (K) 0.5 and 6 hours after P2 irradiation, respectively. L: Quantification of apoptotic cells in Ptch $1^{+/-}$and WT mouse lenses at 6 hours after irradiation. M and N: The p21 and p53 immunoreactivity evaluated 4.5 hours after P2 irradiation. $\mathbf{0}$ and P: Proliferating LECs immunostained with Ki-67 (0) and nestin immunoreactivity in neonatally irradiated mice (P2) at 10 days of age (IR/P10) (P). Q: Kinetics of proliferation of Ptch $1^{+/-}$and WT mouse LECs, quantified at different times after P2 irradiation; for comparison, the kinetics of LECs from unirradiated mice (E) are shown (dashed lines). There was a statistically significant difference versus unirradiated age-matched counterparts at all experimental time points. ${ }^{*} P<0.05$, ${ }^{* *} P<0.01$, and ${ }^{* * * *} P<0.0001$. Scale bars: $10 \mu \mathrm{m}$.

mouse lenses at 21 days of age. The results (averaged on six mice) showed no significant difference in lens area between the two genotypes (Supplemental Figure S1).

Because Shh has been implicated in maintenance of a stem cell condition, ${ }^{23}$ we used an antibody against nestin, a typical marker of epidermal stem cells, ${ }^{24}$ to characterize LECs at P2 and P10. We observed substantial differences in LECs at these postnatal ages. In fact, although all LECs at P2 strongly expressed nestin, independent of localization (central and germinative zone) (Figure 4, F and G), nestin expression was significantly reduced in the centrally located LECs at P10, whereas it remained similarly expressed in the germinative zone (Figure 4, H and I, and Supplemental Figure S2). This result indicates that essentially all LECs at P2 have stem/progenitor cell properties, which then regionally decline with mouse age.
Stem/progenitor cells are defined as the cells that execute the repair of damaged tissue. ${ }^{25}$ To examine the mechanisms behind cataract susceptibility of LECs at P2, we evaluated their DNA damage response at short times after irradiation. By using an antibody against $\gamma-\mathrm{H} 2 \mathrm{AX}$, a marker of double-stranded breaks, we show that virtually all LECs showed many $\gamma$-H2AX foci at 0.5 hours after exposure, independent of Ptchl genotype (Figure 4J). Because double-stranded breaks precede cell death via activation of pro-apoptotic pathways, we performed immunostaining with cleaved caspase- 3 antibody, the main executioner of apoptosis, at 6 hours after irradiation. Unexpectedly, despite widespread DNA damage in LECs, only sporadic caspase-3-positive cells were observed (Figure $4 \mathrm{~K}$ ), resulting in a low apoptotic index (approximately $2.5 \%$ ), with no significant difference between 
A

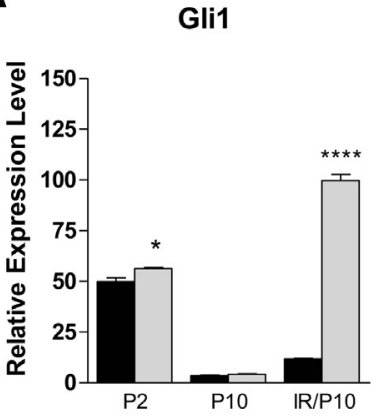

D

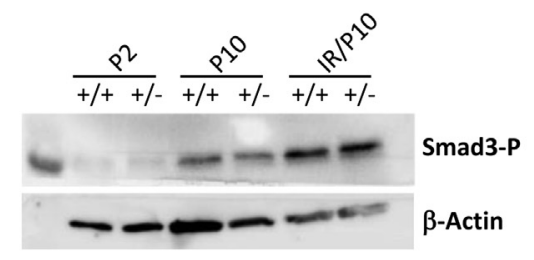

B

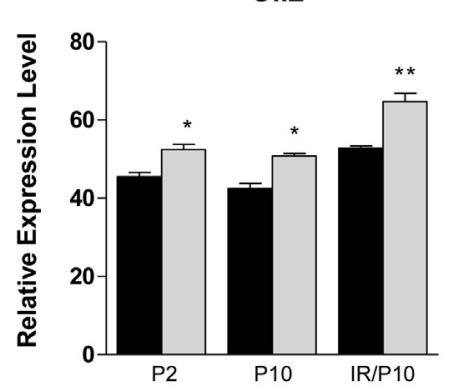

E

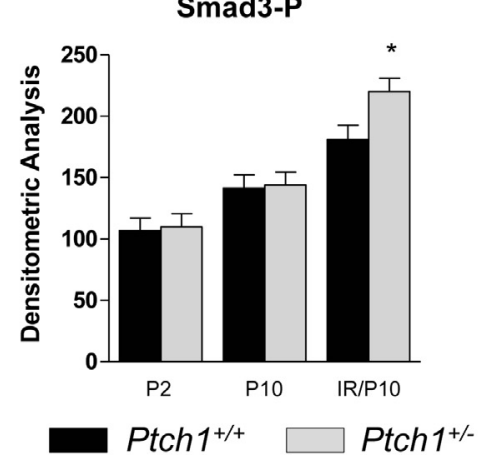

C

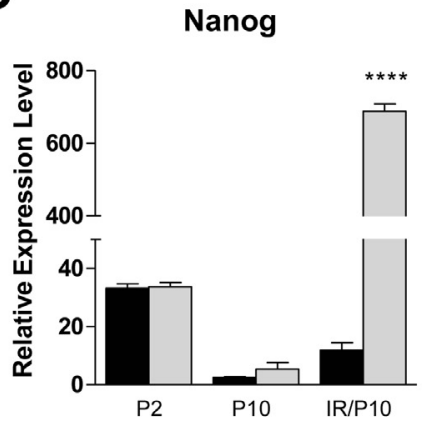

$\mathbf{F}$

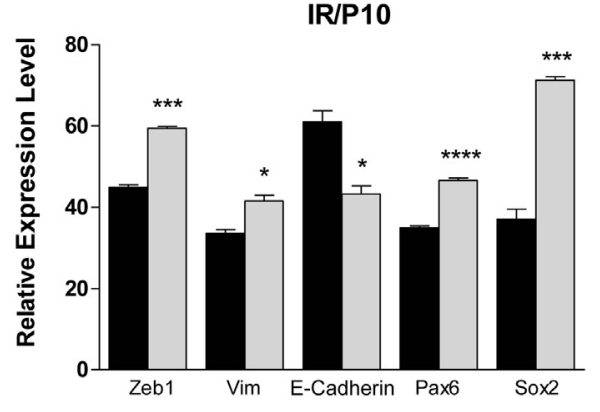

Figure 5 Sonic hedgehog (Shh) and transforming growth factor $\beta$ signaling synergize with radiation damage to promote Nanog overexpression and induce epithelial-to-mesenchymal transition (EMT) in irradiated lens. Relative mRNA expression levels of Gli1 (A), Gli2 (B), and Nanog (C) in unirradiated Ptch1 $1^{+/-}$ and wild-type (WT) unirradiated lens at P2 and P10, and in neonatally exposed mice (P2) at 10 days of age (IR/P10). D: Western blot analysis of phosphorylated Smad3 ( $\mathrm{p}-\mathrm{Smad3}$ ) expression in unirradiated $P t c h 1^{+/-}$and WT lens at P2 and P10, and in IR/P10. E: Band intensities of p-Smad3 were sampled three times and normalized against $\beta$-actin. F: mRNA expression levels of EMT-related markers Zeb1, Vim, and E-cadherin, and stemness genes Pax6 and Sox2 evaluated in IR/P10. ${ }^{*} P<0.05,{ }^{* *} P<0.01,{ }^{* * *} P<0.001$, and ${ }^{* * *} P<0.0001$.

Ptchl $^{+/-}$and WT mice (Figure $4 \mathrm{~L}$ ). The low rate of radiation-induced apoptosis is in agreement with stem/ progenitor cell properties of LECs at P2.

Consistent with the low apoptotic rate after irradiation, we show strong expression of p21 (Figure 4M), which acts as an inhibitor of apoptosis in several systems. ${ }^{26} \mathrm{We}$ also examined $\mathrm{p} 53$ levels in the mouse lens irradiated at P2 and found a moderate p53 expression (Figure $3 \mathrm{~N}$ ). This is consistent with previous results from our group in irradiated neonatal cerebellum, ${ }^{27}$ and with recent findings obtained in different stem cell compartments showing that p53 is not a key player in the DNA damage response of stem cells and that X-rays induce p53-independent up-regulation of $\mathrm{p} 21$, preventing apoptosis and promoting cell cycle entry and expansion of stem cells. ${ }^{28}$

We also analyzed LECs at longer times after P2 irradiation. Independent of genotype, irradiated mice sacrificed at P10 showed more Ki-67-positive LECs compared to unirradiated age-matched counterparts (Figure 4, O and C, respecitvely). Time course analysis at 4 and 6 days confirmed a significantly higher proliferation index in irradiated mice (Figure $4 \mathrm{~N}$ ), regardless of Ptchl allelic status. At 10 days, this difference was significant only in Ptch1 ${ }^{+/-}$mice. Indeed, the proliferation index of LECs from Ptchl ${ }^{+/-}$mice was significantly higher compared to WT mice at all time points analyzed. Finally, we examined whether increased proliferation in irradiated LECs correlated with nestin expression. ${ }^{29}$ Notably, higher levels of nestin were detected at P10 (Figure 4O) compared to age-matched unirradiated mice (Figure $4 \mathrm{H}$ ), resembling nestin expression at P2 (Figure 4F). Supplemental Figure $\mathrm{S} 1$ provides quantification.

The increased rate of proliferation and the higher expression of nestin suggest that radiation damage may stimulate recruitment into the cell cycle and prevent exhaustion of LEC self-renewal competence after irradiation at P2 in both Ptchl $^{+/-}$and WT mice. Nevertheless, the strong increase of cataract development only in exposed Ptch ${ }^{+/-}$mice supports the hypothesis that additional mechanisms are involved in $P t c h 1^{+/-}$LEC radiosensitivity.

Cross Talk between Shh and TGF- $\beta$ Signaling Pathways in Radiogenic Cataract Development

To test the hypothesis that the age-dependent sensitivity of LECs to irradiation is controlled by Shh-dependent regulation of the stem cell competence of LECs, mRNA levels of Gli1 and Gli2, the downstream effectors of the Shh pathway, were evaluated in unirradiated LECs by qPCR. Results show a high expression of Gli1 at P2 and a sharp decline at P10 (approximately 12-fold; $P<0.0001$ ) (Figure 5A), indicative of Shh pathway inactivity irrespective of Ptch1 status. At P2, Ptch $1^{+/-}$LECs showed a higher Gli1 mRNA level compared 


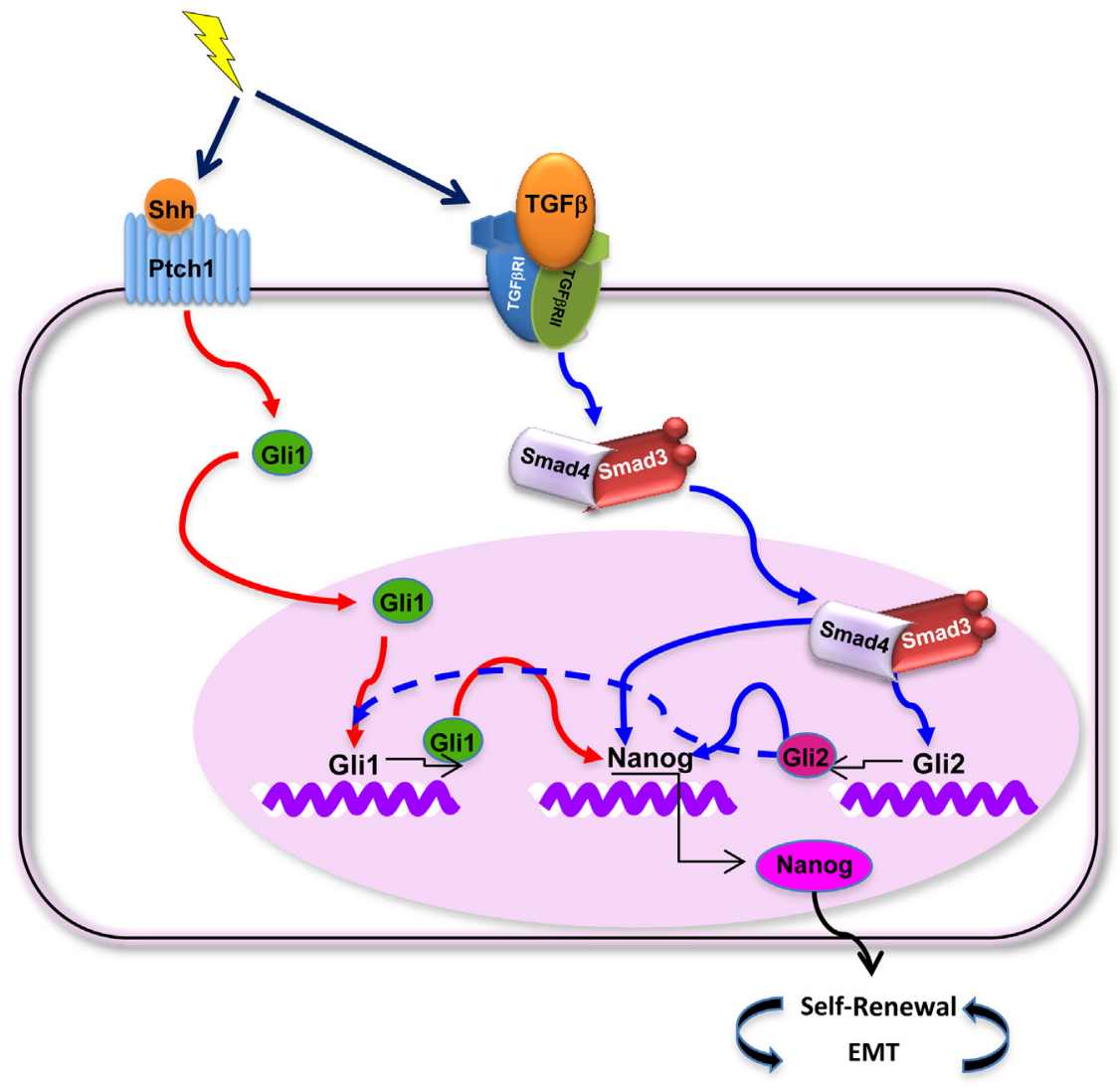

Figure 6 Model of interaction between sonic hedgehog (Shh) and transforming growth factor $\beta$ (TGF- $\beta$ ) signaling in irradiated lens epithelial cells, consistent with in vivo findings from this study. Radiation injury may up-regulate Shh expression directly or indirectly via TGF- $\beta$ signaling. Gli2 is an early gene target of TGF- $\beta /$ Smad signaling, independent of Shh signals, and Gli1 can be induced by TGF- $\beta$ in a Gli2-dependent manner. At P2, both signaling pathways converge on Nanog in Patched $1\left(\right.$ Ptch $\left.^{+/-}\right)$injured lens tissue (blue and red arrows), facilitating a self-amplifying Shh/ TGF- $\beta$ pathogenic loop promoting and amplifying epithelial-to-mesenchymal transition (EMT), and inducing cataract development. When Shh is normally regulated (WT mice at $\mathrm{P} 2$ ) or inactive (P10), the self-amplifying Shh/TGF- $\beta$ pathogenic loop is abrogated, and only indirect activation of Shh signaling via TGF- $\beta$ (blue arrows) contributes to Nanog expression and EMT, resulting in low cataract induction. to WT LECs $(P=0.0364)$, consistent with Shh pathway deregulation in Ptchl $^{+/-}$animals. High Gli2 mRNA levels were observed at P2 in both $P t c h 1^{+/-}$and WT LECs (Figure 5B). Notably, Gli2 expression did not decline at P10, showing Gli2 independence from Shh signaling at this age. Again, Ptch1 ${ }^{+/}$LECs showed significantly higher Gli2 levels relative to WT LECs at both ages $(\mathrm{P} 2, P=0.0454$; P10, $P=0.0165$ ).

It is known that Shh signaling regulates Nanog expression in different systems. ${ }^{30,31}$ To determine whether Nanog could play a role in age-related cataract induction, we measured its mRNA levels at P2 and P10. Nanog mRNA decreased sharply during this time window $(P<0.0001)$, consistent with the hypothesis of stemness rapid decline in mouse LECs during lens postnatal development (Figure 5C).

A common feature of ASC is induction of EMT by TGF- $\beta,{ }^{32,33}$ and cross talk between Shh signals and TGF- $\beta$ in different pathological conditions has been observed. ${ }^{34-36}$ Therefore, it was important to assess the balance of Shh and TGF- $\beta$ signaling during postnatal lens development with or without radiation insult. Because TGF- $\beta$ signals through phosphorylation of the Smad3 transcription factor of the TGF$\beta$ superfamily ligands, we investigated expression of phosphorylated Smad3 (p-Smad3). Western blot analysis results show a trend toward increased $\mathrm{p}-\mathrm{Smad} 3$ expression from $\mathrm{P} 2$ to P10. Moreover, p-Smad3 expression was not significantly different between $P t c h 1^{+/-}$and WT LECs (Figure 5, C and D).
Expression levels of Shh and TGF- $\beta$ are known to increase after radiation injury. ${ }^{37,38} \mathrm{We}$, therefore, evaluated the expression of Gli1 and Gli2 mRNAs and of p-Smad3 protein at P10, 8 days after irradiation. Gli1 mRNA was upregulated relative to LECs from age-matched unirradiated mice (Figure 5A). Remarkably, Ptch1 ${ }^{+/-}$LECs showed a 24-fold increase of Gli1 expression after radiation injury $(P<0.0001)$ compared with only a 3.5 -fold increase in WT LECs $(P<0.0001)$. Relative to Gli1, Gli2 mRNA expression was only marginally increased (approximately 1.3fold), again with significantly higher levels in $P t c h 1^{+-}$ mice (Figure 5B). p-Smad3 protein was also significantly up-regulated in irradiated Ptch1 ${ }^{+/-}$mice (Figure 5, D and E), showing differential activation of TGF- $\beta /$ Smad pathway signaling.

A trait of ASC formation is EMT of LECs into myofibroblasts. ${ }^{33}$ To determine whether irradiated $\mathrm{Ptchl}^{+/-}$and WT LECs show differences in markers associated with EMT, we examined, by qPCR, the expression of EMTrelated genes Zeb1 and Vim. ${ }^{39,40}$ Figure $5 \mathrm{~F}$ shows significantly increased expression of the EMT regulator Zeb1 and the mesenchymal marker Vim in irradiated Ptchl ${ }^{+/-}$LECs. Moreover, expression of the cell adhesion molecule E-cadherin was significantly lower in LECs from $\mathrm{Ptchl}^{+/-}$irradiated mice, consistent with increased expression of mesenchymal markers. These results suggest that interaction of the Shh and TGF- $\beta /$ Smad signaling pathways can induce 
EMT and promote fibrogenesis in radiation-injured $P t c h 1^{+/-}$ mouse lens.

Cells that undergo EMT are known to acquire stem cell-like properties. ${ }^{41} \mathrm{We}$, therefore, evaluated mRNA levels of Pax6 and Sox 2 stem cell markers in irradiated WT and $P t c h 1^{+/-}$LECs. Indeed, we found the expression levels of these stemness-associated genes significantly increased in Ptch $^{+/-}$compared to WT mice. Finally, analysis of Nanog expression after $\mathrm{P} 2$ irradiation revealed a striking increase (approximately 130-fold) in Ptchl ${ }^{+/-}$LECs at P10 $(P<0.0001)$ (Figure 5C), suggesting a central role of Nanog in Ptchl-associated cataractogenesis.

\section{Discussion}

Although Gorlin syndrome patients, who have a deregulated Shh pathway, are prone to ophthalmic abnormalities, including cataract, a role for Shh in cataract development has not been previously reported. We establish herein that mice heterozygous for the Ptchl gene, the Shh receptor, develop spontaneous cataract and are more sensitive than WT animals to the cataractogenic effects of ionizing radiation. The mechanisms of cataract formation are linked to DNA damage of LECs, which can result in defective cell cycle control and fibrotic reaction. ${ }^{42} \mathrm{Shh}$ is involved in both processes, and as our results show, animals deficient in Ptchl protein are susceptible to cataract induction.

A common response to DNA damage in differentiated cells is activation of p53 and increased expression of p21 cyclin-dependent kinase inhibitor to arrest the cell cycle. However, we show that LECs irradiated at P2 evade apoptosis and proliferation arrest, suggesting a nonfunctional p53-p21 axis of the $\mathrm{G}_{1} / \mathrm{S}$ checkpoint pathway, ${ }^{28}$ and further strengthening the hypothesis that the lens epithelium includes a true stem cell population. ${ }^{43}$

We show that radiation-induced cataractogenesis is subjected to temporal restriction. Although early studies have shown age-dependent responses to radiation, ${ }^{44}$ the underlying mechanisms have remained elusive. In the rodent lens, the most rapid growth occurs during the first postnatal week, with increases in lens weight of up to $87 \%$ in a single day. ${ }^{45}$ In principle, because radiosensitivity is higher in proliferating cells, ${ }^{46}$ the temporal restriction observed might be attributable to age-related decline of proliferation in LECs during postnatal lens development. However, we observe that exposure of $P t c h 1^{+/-}$mice to irradiation at P10 fails to induce cataract, despite evident ongoing proliferation in P10 lens. Likewise, although Ptch1 ${ }^{+/-}$mice have an increased percentage of proliferating cells in their lenses compared to WT mice throughout the first postnatal week, this is unlikely to explain a 5.4-fold cataract incidence in P2-irradiated Ptch1 $1^{+/-}$mice, which indicates that additional factors are likely to influence LEC susceptibility to radiogenic cataract.

It is unclear how Ptchl haploinsufficiency can predispose to spontaneous and radiation-induced cataract development.
The Shh mitogenic role in the developing mammalian lens is recognized, and previous data have shown that patterns of fiber cell differentiation are perturbed in mouse lens with constitutive activation of smoothened, a key signal transducer in the Shh pathway. ${ }^{16}$ However, there is a shortage of data about the Shh role during lens postnatal growth. Indeed, our work did not reveal grossly visible or histological abnormalities in the newborn or adult unirradiated $P t c h 1^{+/-}$ lens, except for spontaneous cataract development in $8.8 \%$ of mice. To explore Shh signaling involvement in lens postnatal growth, we measured Gli1 transcript levels as a readout of Shh pathway activation. Although at P2 we found expression of Gli1, with significantly higher levels in Ptchl $^{+/-}$mice compared to WT littermates, at P10 there was a sharp decrease of Gli1 expression, consistent with the reduction of number of proliferating cells in the germinative zone at this time of lens development. We also show that Gli1 expression was considerably up-regulated by irradiation and that increased Shh signaling correlates with proliferation of progenitor cells, suggesting that the Shh pathway could control mammalian lens regeneration after lens injury. Moreover, the large difference observed between Gli1 levels in exposed Ptchl $^{+/-}$compared to WT mice suggests a causal role for Gli1 in cataract. We note, in addition, that a different expression pattern was evident between Gli1 and Gli2, with Gli2 still highly expressed at P10.

Cross talk between Shh and TGF- $\beta$ signaling in cancer has been recognized. ${ }^{34}$ However, not much is known about its role in driving tissue fibrosis. TGF- $\beta$ is required for embryonic eye development, driving, in particular, lens development and growth by the tight spatial and temporal regulation of lens cell proliferation and fiber cell differentiation. ${ }^{47-49}$ TGF- $\beta$ also plays crucial roles during ocular fibrotic diseases, such as ASC development, in which LECs undergo EMT via TGF- $\beta$ activation. ${ }^{33,42,50-52}$ Indeed, our data show that $\mathrm{p}-\mathrm{Smad} 3$ expression increased both in $P t c h 1^{+/-}$and WT mice, as reflected by $8.8 \%$ cataract incidence in WT mice irradiated at P2. However, TGF- $\beta$ is also a potent inducer of Gli2, and consequently, of Gli1, independent of Shh/Ptch1 signaling, and the cross talk between pathways mediated by Gli2 might increase significantly in mice with hyperactive Shh signaling. ${ }^{34}$ Further descriptive and functional experiments will be required to prove or refute this hypothesis. Although this is something we intend to follow up, it is tempting to speculate that TGF- $\beta / S$ mad signaling might contribute to the expression of Gli2, facilitating a selfamplifying Shh/TGF- $\beta$ pathogenic loop that may promote and amplify the EMT process in the lens after radiation injury. Indeed, interaction between Shh and TGF- $\beta$ signaling has been previously reported in human fibrotic lung disease, ${ }^{35}$ and recent data have shown its role in mediating cyclosporine-enhanced gingival hyperplasia. ${ }^{36}$

More important, a strong correlation was found between Shh deregulation, radiation damage, and expression of Nanog, which was greatly increased only in irradiated $\mathrm{Ptchl}^{+/-}$mice. Although there has been little study on the 
role of Nanog in cataractogenesis, a previous article investigating potential oncogenic functions of Nanog in transgenic mice expressing high levels of the NanogP8 homologue reported development of early cataract with high penetrance in lines expressing higher levels of the transgene. ${ }^{53}$ It is presently unclear how high Nanog expression associates to enhanced cataract development, but unexpected phenotypes observed in K14-NanogP8 mice (eg, perinatal lethality, developmental defects, resistance to two-stage skin carcinogenesis, and wound-healing defects) suggested that high levels of NanogP8 may disrupt normal developmental programs and inhibit oncogenic processes by depleting stem cells. This implies that Nanog expression levels must be tightly controlled for proper development and response to injury. In addition, a recent report found that expression of matrix metalloproteinase- 9 , a direct target of Nanog, ${ }^{54}$ is critical in TGF- $\beta$-induced ASC formation. ${ }^{55}$ Although further studies will be needed to better elucidate Nanog's role in modulating cataractogenesis, there is evidence that Gli1 regulates Nanog expression in different systems. ${ }^{30,31}$ Moreover, TGF- $\beta$ regulates Nanog and promotes EMT in many cancers ${ }^{56,57}$ and in non-cancer-related pathological conditions. ${ }^{58}$ Therefore, in the interplay of Shh and TGF- $\beta$, Nanog might act as a critical player on which both signaling pathways converge in injured lens tissue (Figure 6).

Although Shh signaling is linked with regulation of injury repair and wound healing after tissue damage and with promotion of fibrosis in several tissues, ${ }^{51,59}$ its role in the pathogenesis of lens fibrosis is completely unknown. Results from the current study demonstrate, for the first time, a key role of Shh in spontaneous and radiation-induced cataract development, and suggest a cross talk between Shh and TGF- $\beta$ converging on Nanog. We, in addition, provide a novel powerful animal model to investigate the mechanisms of cataract development. Further studies in this area will be needed because identification of the main molecular mechanisms involved in the induction of EMT and subsequent fibrotic alterations in lens cells may potentially help reduce caractogenic effects after therapeutic radiation exposures, especially in survivors of childhood cancer who are at increased risk for ocular late effects, including cataract.

\section{Supplemental Data}

Supplemental material for this article can be found at http://dx.doi.org/10.1016/j.ajpath.2014.09.019.

\section{References}

1. Foster A, Resnikoff S: The impact of Vision 2020 on global blindness. Eye 2005, 19:1133-1135

2. Robman L, Taylor H: External factors in the development of cataract. Eye (Lond) 2005, 19:1074-1082

3. Gilbert C, Foster A: Childhood blindness in the context of VISION 2020-The Right to Sight. Bull World Health Organ 2001, 79:227-232
4. Ferrufino-Ponce ZK, Henderson BA: Radiotherapy and cataract formation. Semin Ophthalmol 2006, 21:171-180

5. Gorlin RJ: Nevoid basal cell carcinoma syndrome. Dermatol Clin 1995, 13:113-125

6. Saran A: Basal cell carcinoma and the carcinogenic role of aberrant Hedgehog signaling. Future Oncol 2010, 6:1003-1014

7. O'Malley S, Weitman D, Olding M, Sekhar L: Multiple neoplasms following craniospinal irradiation for medulloblastoma in a patient with nevoid basal cell carcinoma syndrome: case report. J Neurosurg 1997, 86:286-288

8. Evans DG, Ladusans EJ, Rimmer S, Burnell LD, Thakker N, Farndon PA: Complications of the naevoid basal cell carcinoma syndrome: results of a population based study. J Med Genet 1993, 30:460-464

9. Taylor SF, Cook AE, Leatherbarrow B: Review of patients with basal cell nevus syndrome. Ophthal Plast Reconstr Surg 2006, 22:259-265

10. Hahn H, Wojnowski L, Zimmer AM, Hall J, Miller G, Zimmer A Rhabdomyosarcomas and radiation hypersensitivity in a mouse model of Gorlin syndrome. Nat Med 1998, 4:619-622

11. Mancuso M, Pazzaglia S, Tanori M, Hahn H, Merola P, Rebessi S, Atkinson MJ, Di Majo V, Covelli V, Saran A: Basal cell carcinoma and its development: insights from radiation-induced tumors in Ptch1deficient mice. Cancer Res 2004, 64:934-941

12. Pazzaglia S, Mancuso M, Atkinson MJ, Tanori M, Rebessi S, Majo VD, Covelli V, Hahn H, Saran A: High incidence of medulloblastoma following X-ray-irradiation of newborn Ptc1 heterozygous mice. Oncogene 2002, 21:7580-7584

13. Barakat MT, Humke EW, Scott MP: Learning from Jekyll to control Hyde: hedgehog signaling in development and cancer. Trends Mol Med 2010, 16:337-348

14. Boulton M, Albon J: Stem cells in the eye. Int J Biochem Cell Biol 2004, 36:643-657

15. Zhou M, Leiberman J, Xu J, Lavker RM: A hierarchy of proliferative cells exists in mouse lens epithelium: implications for lens maintenance. Invest Ophthalmol Vis Sci 2006, 47:2997-3003

16. Kerr CL, Huang J, Williams T, West-Mays JA: Activation of the hedgehog signaling pathway in the developing lens stimulates ectopic FoxE3 expression and disruption in fiber cell differentiation. Invest Ophthalmol Vis Sci 2012, 53:3316-3330

17. Tsonis PA, Vergara MN, Spence JR, Madhavan M, Kramer EL, Call MK, Santiago WG, Vallance JE, Robbins DJ, Del Rio-Tsonis K A novel role of the hedgehog pathway in lens regeneration. Dev Biol 2004, 267:450-461

18. Mancuso M, Pasquali E, Leonardi S, Rebessi S, Tanori M, Giardullo P, Borra F, Pazzaglia S, Naus CC, Di Majo V, Saran A: Role of connexin43 and ATP in long-range bystander radiation damage and oncogenesis in vivo. Oncogene 2011, 30:4601-4608

19. Beebe DC, Vasiliev O, Guo J, Shui YB, Bassnett S: Changes in adhesion complexes define stages in the differentiation of lens fiber cells. Invest Ophthalmol Vis Sci 2001, 42:727-734

20. Lovicu FJ, McAvoy JW: Spatial and temporal expression of p57(KIP2) during murine lens development. Mech Dev 1999, 86:165-169

21. Sellitto C, Li L, White TW: Connexin50 is essential for normal postnatal lens cell proliferation. Invest Ophthalmol Vis Sci 2004, 45: 3196-3202

22. Wechsler-Reya RJ, Scott MP: Control of neuronal precursor proliferation in the cerebellum by Sonic Hedgehog. Neuron 1999, 22:103-114

23. Reya T, Morrison SJ, Clarke MF, Weissman IL: Stem cells, cancer, and cancer stem cells. Nature 2001, 414:105-111

24. Watarai A, Amoh Y, Maejima H, Hamada Y, Katsuoka K: Nestin expression is increased in the suprabasal epidermal layer in psoriasis vulgaris. Acta Derm Venereol 2013, 93:39-43

25. Hoffmann A, Tsonis PA: Pattern formation in regenerating tissues. Edited by Capasso V, Gromov M, Harel-Bellan A, Morozova N, Pritchard LL. In Pattern Formation in Morphogenesis, Springer Proceedings in Mathematics, Vol. 15. Springer-Verlag Berlin Heidelberg, 2013, pp 7-15

26. Gartel AL, Tyner AL: The role of the cyclin-dependent kinase inhibitor p21 in apoptosis. Mol Cancer Ther 2002, 1:639-649 
27. Pazzaglia S, Tanori M, Mancuso M, Rebessi S, Leonardi S, Di Majo V, Covelli V, Atkinson MJ, Hahn H, Saran A: Linking DNA damage to medulloblastoma tumorigenesis in patched heterozygous knockout mice. Oncogene 2006, 25:1165-1173

28. Insinga A, Cicalese A, Faretta M, Gallo B, Albano L, Ronzoni S, Furia L, Viale A, Pelicci PG: DNA damage in stem cells activates $\mathrm{p} 21$, inhibits $\mathrm{p} 53$, and induces symmetric self-renewing divisions. Proc Natl Acad Sci U S A 2013, 110:3931-3936

29. Xue XJ, Yuan XB: Nestin is essential for mitogen-stimulated proliferation of neural progenitor cells. Mol Cell Neurosci 2010, 45:26-36

30. Po A, Ferretti E, Miele E, De Smaele E, Paganelli A, Canettieri G, Coni S, Di Marcotullio L, Biffoni M, Massimi L, Di Rocco C, Screpanti I, Gulino A: Hedgehog controls neural stem cells through p53-independent regulation of Nanog. EMBO J 2010, 29:2646-2658

31. Zbinden M, Duquet A, Lorente-Trigos A, Ngwabyt SN, Borges I, Ruiz i Altaba A: NANOG regulates glioma stem cells and is essential in vivo acting in a cross-functional network with GLI1 and p53. EMBO J 2010, 29:2659-2674

32. de Iongh RU, Wederell E, Lovicu FJ, McAvoy JW: Transforming growth factor-beta-induced epithelial-mesenchymal transition in the lens: a model for cataract formation. Cells Tissues Organs 2005, 179:43-55

33. Nathu Z, Dwivedi DJ, Reddan JR, Sheardown H, Margetts PJ, WestMays JA: Temporal changes in MMP mRNA expression in the lens epithelium during anterior subcapsular cataract formation. Exp Eye Res 2009, 88:323-330

34. Javelaud D, Pierrat MJ, Mauviel A: Crosstalk between TGF-beta and hedgehog signaling in cancer. FEBS Lett 2012, 586:2016-2025

35. Stewart GA, Hoyne GF, Ahmad SA, Jarman E, Wallace WA, Harrison DJ, Haslett C, Lamb JR, Howie SE: Expression of the developmental Sonic hedgehog (Shh) signalling pathway is upregulated in chronic lung fibrosis and the Shh receptor patched 1 is present in circulating T lymphocytes. J Pathol 2003, 199:488-495

36. Chung Y, Fu E: Crosstalk between Shh and TGF-beta signaling in cyclosporine-enhanced cell proliferation in human gingival fibroblasts. PLoS One 2013, 8:e70128

37. Ma J, Tian L, Cheng J, Chen Z, Xu B, Wang L, Li C, Huang Q: Sonic hedgehog signaling pathway supports cancer cell growth during cancer radiotherapy. PLoS One 2013, 8:e65032

38. Dancea HC, Shareef MM, Ahmed MM: Role of radiation-induced TGFbeta signaling in cancer therapy. Mol Cell Pharmacol 2009, 1:44-56

39. Liu Y, El-Naggar S, Darling DS, Higashi Y, Dean DC: Zeb1 links epithelial-mesenchymal transition and cellular senescence. Development 2008, 135:579-588

40. Ivaska J: Vimentin: central hub in EMT induction? Small GTPases 2011, 2:51-53

41. Mani SA, Guo W, Liao MJ, Eaton EN, Ayyanan A, Zhou AY, Brooks M, Reinhard F, Zhang CC, Shipitsin M, Campbell LL, Polyak K, Brisken C, Yang J, Weinberg RA: The epithelialmesenchymal transition generates cells with properties of stem cells. Cell 2008, 133:704-715

42. Shin EH, Basson MA, Robinson ML, McAvoy JW, Lovicu FJ: Sprouty is a negative regulator of transforming growth factor $\beta$-induced epithelial-to-mesenchymal transition and cataract. Mol Med 2012, 18:861-873

43. Oka M, Toyoda C, Kaneko Y, Nakazawa Y, Aizu-Yokota E, Takehana M: Characterization and localization of side population cells in the lens. Mol Vis 2010, 16:945-953
44. Gajewski AK, Majewska K, Slowikowska MG, Chomiczewski K, Kulig A: Types and rate of cataract development in mice irradiated at different ages. Radiat Res 1977, 71:471-480

45. Brewitt B, Clark JI: Growth and transparency in the lens, an epithelial tissue, stimulated by pulses of PDGF. Science 1988, 242:777-779

46. Stobbe CC, Park SJ, Chapman JD: The radiation hypersensitivity of cells at mitosis. Int J Radiat Biol 2002, 78:1149-1157

47. de Iongh RU, Lovicu FJ, Overbeek PA, Schneider MD, Joya J, Hardeman ED, McAvoy JW: Requirement for TGFbeta receptor signaling during terminal lens fiber differentiation. Development 2001, 128:3995-4010

48. Beebe D, Garcia C, Wang X, Rajagopal R, Feldmeier M, Kim JY, Chytil A, Moses H, Ashery-Padan R, Rauchman M: Contributions by members of the TGFbeta superfamily to lens development. Int J Dev Biol 2004, 48:845-856

49. Rajagopal R, Dattilo LK, Kaartinen V, Deng CX, Umans L, Zwijsen A, Roberts AB, Bottinger EP, Beebe DC: Functions of the type 1 BMP receptor Acvr1 (Alk2) in lens development: cell proliferation, terminal differentiation, and survival. Invest Ophthalmol Vis Sci 2008, 49:4953-4960

50. Saika S, Kono-Saika S, Ohnishi Y, Sato M, Muragaki Y, Ooshima A, Flanders KC, Yoo J, Anzano M, Liu CY, Kao WW, Roberts AB: Smad3 signaling is required for epithelial-mesenchymal transition of lens epithelium after injury. Am J Pathol 2004, 164:651-663

51. Banh A, Deschamps PA, Gauldie J, Overbeek PA, Sivak JG, WestMays JA: Lens-specific expression of TGF-beta induces anterior subcapsular cataract formation in the absence of Smad3. Invest Ophthalmol Vis Sci 2006, 47:3450-3460

52. Lovicu FJ, Schulz MW, Hales AM, Vincent LN, Overbeek PA, Chamberlain CG, McAvoy JW: TGFbeta induces morphological and molecular changes similar to human anterior subcapsular cataract. $\mathrm{Br}$ J Ophthalmol 2002, 86:220-226

53. Badeaux MA, Jeter CR, Gong S, Liu B, Suraneni MV, Rundhaug J, Fischer SM, Yang T, Kusewitt D, Tang DG: In vivo functional studies of tumor-specific retrogene NanogP8 in transgenic animals. Cell Cycle 2013, 12:2395-2408

54. Siu MK, Wong ES, Chan HY, Ngan HY, Chan KY, Cheung AN: Overexpression of NANOG in gestational trophoblastic diseases: effect on apoptosis, cell invasion, and clinical outcome. Am J Pathol 2008, 173:1165-1172

55. Dwivedi DJ, Pino G, Banh A, Nathu Z, Howchin D, Margetts P, Sivak JG, West-Mays JA: Matrix metalloproteinase inhibitors suppress transforming growth factor-beta-induced subcapsular cataract formation. Am J Pathol 2013, 168:69-79

56. Sun C, Sun L, Jiang K, Gao DM, Kang XN, Wang C, Zhang S, Huang S, Qin X, Li Y, Liu YK: NANOG promotes liver cancer cell invasion by inducing epithelial-mesenchymal transition through NODAL/SMAD3 signaling pathway. Int J Biochem Cell Biol 2013, 45:1099-1108

57. Santaliz-Ruiz LE, Xie X, Old M, Teknos TN, Pan Q, James AG, Solove RJ: Emerging role of nanog in tumorigenesis and cancer stem cells. Int J Cancer 2014, 135:2741-2748

58. Bi WR, Yang CQ, Shi Q: Transforming growth factor- $\beta 1$ induced epithelial-mesenchymal transition in hepatic fibrosis. Hepatogastroenterology 2012, 59:1960-1963

59. Greenbaum LE: Hedgehog signaling in biliary fibrosis. J Clin Invest 2008, 118:3263-3265 